\title{
Effect of frequency difference on sensitivity of beats perception
}

\author{
Soo-Chul Lim $\cdot$ Ki-Uk Kyung $\cdot$ Dong-Soo Kwon
}

Received: 8 June 2010 / Accepted: 3 September 2011 / Published online: 25 October 2011

(C) The Author(s) 2011. This article is published with open access at Springerlink.com

\begin{abstract}
Two vibrations with slightly different frequencies induce the beats phenomenon. In tactile perception, when two pins of different frequencies stimulate the fingertips, an individual perceives a beats caused by a summation stimulus of the two vibrations. The present study demonstrates experimentally that humans can perceive another vibration based on the beats phenomenon when two tactile stimuli with slightly different frequencies are stimulated on the finger pad with a small contactor in different locations at the same time. Moreover, we examined the amplitude of the detection threshold to be able to perceive beats phenomenon on the index finger with 5 carrier frequency (63.1, $100,158.5,251.2$, and $398.1 \mathrm{~Hz}$ ) and 4 beats frequency $(2.5,3.98,6.31$, and $10 \mathrm{~Hz})$ when two stimuli $1 \mathrm{~mm}$ distance apart are vibrated at a slightly different frequency. From the experiments, it is concluded that the amplitude threshold to be able to perceive beats decreases as the standard frequency increases under $398 \mathrm{~Hz}$. Furthermore, from comparing the absolute detection threshold and beats detection threshold, as the carrier frequency increases, the required amplitude at two pins for the detection of beats decreases compared to absolute vibration.
\end{abstract}

This work has been supported by the S/W Computing R\&D program of MKE/KEIT (10035360, TAXEL: Visio-haptic Display and Rendering Engine).

S.-C. Lim · D.-S. Kwon ( $\square)$

Human Robot Interaction Research Center,

KAIST, Daejeon, Korea

e-mail: kwonds@kaist.ac.kr

URL: http://robot.kaist.ac.kr

K.-U. Kyung

Department of Next Generation Computing Research,

ETRI, Daejeon, Korea
Keywords Beats $\cdot$ Vibrotactile $\cdot$ Tactile feedback . Frequency difference $\cdot$ Threshold

\section{Introduction}

Beyond visual and auditory displays, tactile interface is being considered as a new interaction interface that is expected to provide more realistic user experience. The function of tactile displays is to mimic particular features of real surfaces, such as roughness, texture, and thermal conductivity. Mechanisms with multiple stimulators to simulate the surface features have been suggested for the implementation of the tactile displays. Fingertip tactile displays with dense arrangements of pins have been built by various research groups (Frisken-Gibson et al. 1987; Hayward and Cruz-Hernandez 2000; Killebrew et al. 2007; Kyung et al. 2007). When these tactile arrays of pins stimulate the skin, various surface patterns can be presented (Oyarzabal et al. 2007; Lim et al. 2011).

Although the use of multiple pins offers the advantage of stimulating various surface properties, unexpected phenomena are sometimes observed. One example is "the beats." It has been observed that the simultaneous operation of two stimuli with slightly different frequencies causes a new harmonic wave of two tones (von Bekesy 1957, 1959). This wave, termed beats, is caused by the interference between two periodic waves with frequencies $f$ and $f+\delta f$ close to one another (Rao 1995; von Bekesy 1959). The wave is observed as a very low frequency of $\delta f$ and can be felt by the human tactile or auditory sense as if there is another stimulation. The beats' characteristics have been studied in auditory perception research area for a long time; otherwise, their characteristics in tactile perception have been investigated by a small number of research groups. After 
Bekesy's initial study using a loudspeaker for generating vibration, advances in stimulation devices in the terms of precision and wider bandwidth have contributed to observing new beats phenomena in the tactile sense. A recent study used 2 small linear vibrators with different frequency, and each vibrator was held by each hand of the subject. The study observed and measured the new vibration that occurred when two vibrators are directly contacted (Lim et al. 2010). The beats in the tactile sense were also observed when two vibrators are attached to a rigid body (Park and Choi 2011). Another study used unperceivable frequencies in the tactile sense Makino et al. 2011). They used two piezovibrators with very high frequencies over $1 \mathrm{kHz}$, and their frequency difference was $100 \mathrm{~Hz}$. The experimental results showed that the human subject felt the beats frequency of $100 \mathrm{~Hz}$ but could not detect vibration when a single vibration was provided.

The beats in tactile senses are observed in many cases, and they can be designed scientifically and potentially exploited in various applications. In order to use beats as a new methodology for displaying tactile properties, scientific and systematic analysis of the beats phenomenon is required. This study investigated the beats phenomenon in tactile senses on a finger pad using stimulation by two indentation pins with slightly different sinusoidal operation frequencies. We also investigated the beats detection threshold in the tactile sense. We designed a tactile stimulation device with precisely controlled pins and measured the amplitude threshold for the perceived beats sensation when the two stimulus pins were separated by a distance of $1 \mathrm{~mm}$. The stimulation was generated with piezoelectric bimorphs that can be controlled precisely by variation of the frequency and amplitude of the waveform control signal, and we measured the exact displacement of the pins with a laser vibrometer. We term the new stimulation induced after the summation tactile beats, which can be defined as the beats perceived via the tactile sense with two vibratory stimuli presented simultaneously.

\section{Method}

\section{Participants}

Twelve young subjects, aged 23 to 33 years, participated in the experiments. They were all right-handed and reported no known cutaneous or kinesthetic sensing problems. In this research, only participants younger than 33 years of age were selected for all experiments due to the known decreasing sensitivity of elderly people (Dinse et al. 2006; Manning and Tremblay 2006). All the subjects were paid for their participation and were unaware as to the purposes of the experiments.

\section{Apparatus}

Figure 1 shows a schematic diagram of the vibration measurement system. Two pins attached to the piezobimorphs provided external sinusoidal tactile stimuli directly to the index finger. The contact area of the pin was $0.79 \mathrm{~mm}^{2}$ (pin's diameter: $1 \mathrm{~mm}$ ). A laser doppler vibrometer (Keyence LC2400A (controller), LC-2430 (head)) was fixed under the bimorphs to measure the vibration amplitude and frequency. The resolution of the laser doppler vibrometer was less than $0.02 \mu \mathrm{m}$.

Vibrotactile stimuli were delivered through two piezoelectric bimorphs. We used piezoelectric bimorphs (401,055, APC Int. Ltd., PA) with a maximum deflection greater than $700 \mu \mathrm{m}$ and a bandwidth of approximately $350 \mathrm{~Hz}$. Its exerting force was $0.06 \mathrm{~N}$. This force magnitude can sufficiently create an indentation of $0.3 \mathrm{~mm}$ to a human finger pad. The size of the bimorph was $35 \times 2.5 \times 0.6 \mathrm{~mm}$. Voltage amplifiers (PB51, Apex Microtechnology) were used to amplify the circuit so as to obtain a wide supply voltage range $( \pm 15$ to $\pm 150 \mathrm{~V})$. We control piezoelectric bimorphs based on their characteristics like frequency response discovered from our previous work (Kyung et al. 2006).
Fig. 1 Schematic diagram of measuring the piezobimorph amplitude

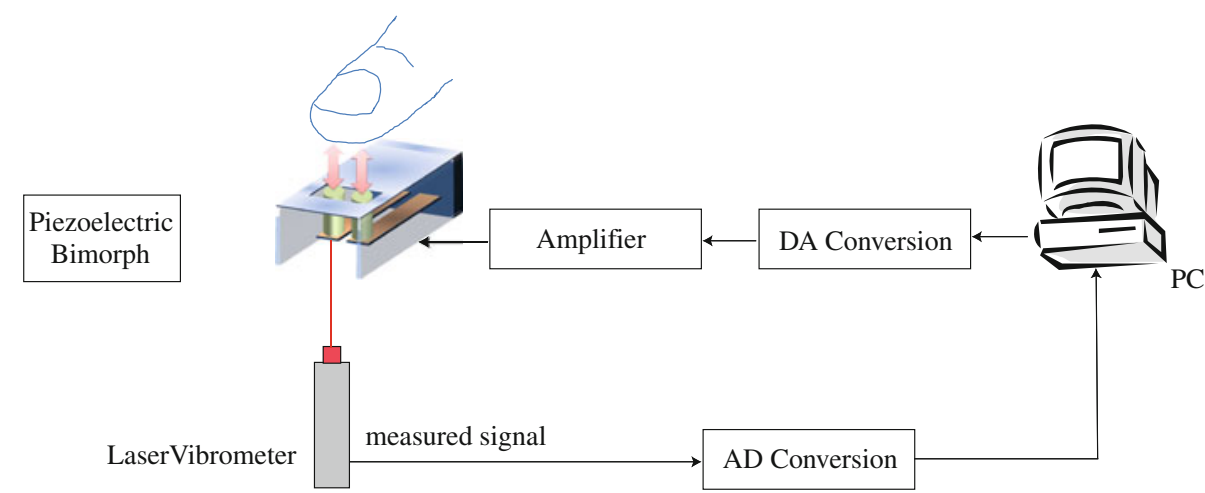


Fig. 2 a Stimulus arrangement b Condition 1: Time chart of stimulus pair between standard vibration and comparison vibration, which is generated by changing the frequency of two pins c Condition 2: Time chart of stimulus pair comparing the standard vibration and comparison vibration, which generates the beats phenomenon just by changing the frequency of Pin2 d Condition 3: Time chart of stimulus pair comparing the standard vibration and comparison vibration, which are vibrated at the same frequency

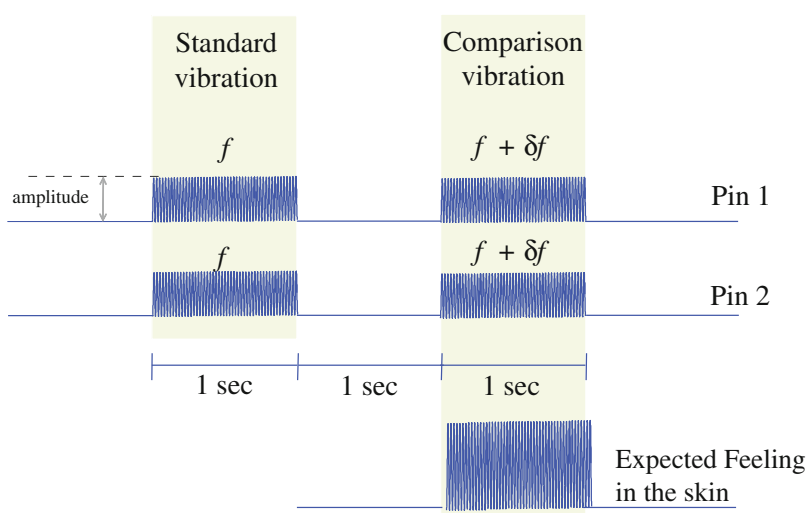

(b)

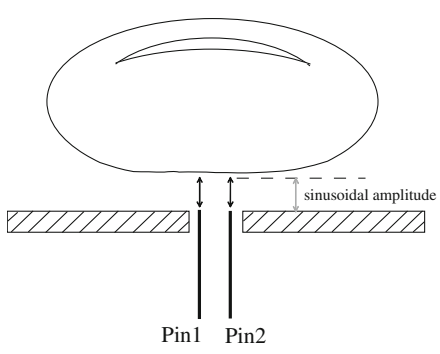

(a)

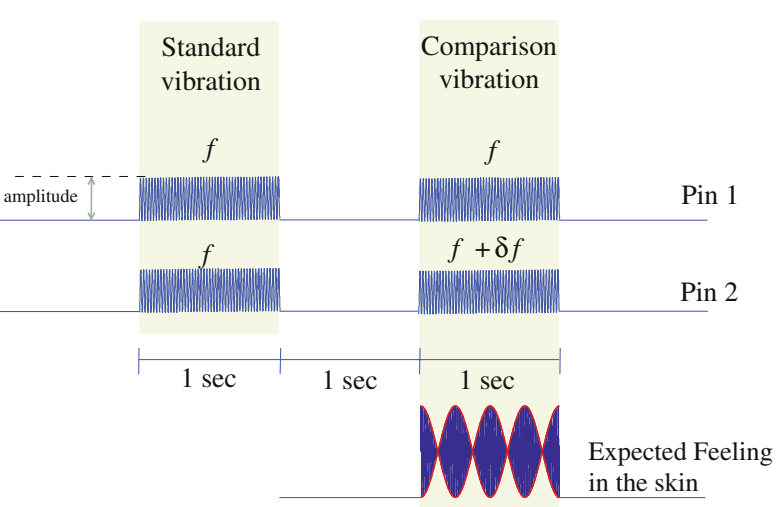

(c)

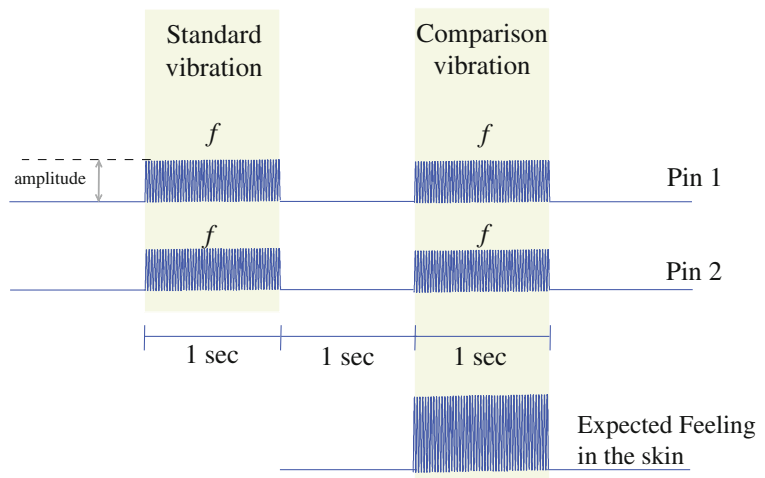

(d)

pair of stimuli (a standard vibration) with another pair (a comparison vibration). The stimuli were generated when using two pins placed at $1 \mathrm{~mm}$ spatial intervals through vibration as shown in Fig. 2b-d.

The participants were seated in front of the tactile stimulus device and instructed to hold their finger, hand, and arm so as to put the finger naturally on the device, so that it can minimize the modulation of sensation from their body position (Medina and Rapp 2008). Participants answered
This experiment was designed to see whether one can sense tactile beats sensation when two pins are stimulated on the finger pad with a slightly different frequency. The design of Experiment 1 is described in Fig. 2. This experiment involved a discrimination test. Participants compared one 
"same" or "different" with two alternative forced choices to compare the standard and comparison vibration in the aspect of tactile sensation on the finger pad. All participants were instructed to say "different" if they were reasonably sure that the comparison vibration was different from the standard vibration in the aspect of tactile sensation and "same" if they could not detect any difference between the standard and comparison vibration. If participants perceived a difference between the standard and the comparison vibration, they are presented with the same stimuli and comparison vibration once more. And then participants report the perceived number of impacts during one second of comparison vibration via the numerical keypad on the keyboard, effectively allowing them to report any pulse number.

Condition 1 of Experiment 1 is shown in Fig. 2b. Two pins vibrate with the same amplitude and frequency in the standard vibration set. The standard frequency $(f)$ is $250 \mathrm{~Hz}$ and amplitude is $40 \mu \mathrm{m}$ in standard vibration of condition 1 . In the comparison vibration set of condition 1 , the amplitude and frequency of two pins are same. However, the frequencies of two pins are slightly different $(f+d f)$ compared with standard vibration. The modulated frequency $(d f)$ is $4 \mathrm{~Hz}$ in comparison vibration of condition 1. Some previous research through experiments has found just noticeable difference (JND) around $250 \mathrm{~Hz}$ in human tactile sensation, while the difference of $4 \mathrm{~Hz}$ is not enough to notice around $250 \mathrm{~Hz}$ (Goff 1967; Mahns et al. 2006; Pongrac 2008). From the previous research, we expect that participants cannot discriminate between standard vibration of $250 \mathrm{~Hz}$ and comparison vibration of $254 \mathrm{~Hz}$ in condition 1 of Experiment 1. Condition 2 of Experiment 1 is shown in Fig. 2c. The standard vibration is the same as condition 1 . In the comparison vibration set of condition 2, frequency of just one pin is modulated with $4 \mathrm{~Hz}$ change. From the similarity between human auditory and tactile system about frequency modulation, we expect that participants can discriminate between the standard vibration of $250 \mathrm{~Hz}$ and comparison vibration of just one pin's modulation to $254 \mathrm{~Hz}$ in condition 2 of Experiment 1 because of beats phenomenon. The condition 3 of Experiment 1 is shown in Fig. 2d. The standard vibration and comparison vibration have the exactly same frequency and amplitude. A random order was employed to present condition 1, 2, and 3 in Fig. 2. Each experiment consisted of ten trials.

To mask any external disturbances, such as the sound and friction noise of the vibrotactile device, pink noise was presented through a noise cancelation headphone. Subjects performed experiments with their eyes open to maintain their arousal level. Between each set of five-minute experiments, the subjects took one-minute breaks to return the sensation in the index fingertip to normal.

\section{Experiment 2: threshold of tactile beats}

This experiment sought to find the amplitude threshold of the two protrusions on the finger pad to detect beats. Two alternative forced choices (same or different) were used to evaluate the beats detection amplitude to stimulate the same methods as those in condition 2 of Experiment 1. We tested at the five standard frequencies $(f)$, which were 63.1, 100, $158.5,251.2$, and $398.1 \mathrm{~Hz}$. The selected standard frequencies are equally spaced on the logarithmic frequency scale. We measured the beats detection threshold just by changing the frequency of Pin2 at the comparison vibration of the four beats frequencies $(\delta f): 2.51,3.98,6.31$, and $10 \mathrm{~Hz}$, at each standard frequency. The test amplitudes at each standard and beats frequencies were presented with one of six predetermined amplitudes, which were determined based on pilot test results. The selected amplitudes are equally spaced on the logarithmic amplitude scale. From pilot studies, we found the rough amplitude thresholds for the beats perception threshold using the discrimination test while changing the stimuli amplitude at each standard and beats frequency using the pattern of condition 2 of Experiment 1. Table 1 shows the amplitude in using the test at each standard frequency $(f)$. For randomly repeated measurements, all stimuli were presented ten times with a uniform random distribution. The subjects' summed responses of "different" were plotted against the amplitude of the two pins at each standard and beats frequency as a psychometric function fitted using sigmoid. In the current study, the beats detection threshold was defined as $75 \%$ in the case of "same" and "different" alternative forced choice results, which is halfway between perfect performance and chance performance.

For within subjects experiment, the stimulus on each beats and standard frequency was presented randomly with all six amplitudes set in advance. Each subject performed a total of 1,250 trials ( 5 beats conditions $\times 5$ standard frequency conditions $\times 6$ amplitude conditions $\times 10$ trials).

Table 1 Test amplitude at each standard and beats frequency

\begin{tabular}{lll}
\hline $\begin{array}{l}\text { Standard } \\
\text { frequency } \\
(\mathrm{Hz})\end{array}$ & $\begin{array}{l}\text { Beats } \\
\text { frequency } \\
(\mathrm{Hz})\end{array}$ & Test amplitude $(\mu \mathrm{m})$ \\
\hline 63.1 & $2.5,3.98,6.31,10$ & $51.3,64.7,81.4,102.5,129,162.5$ \\
100 & $2.5,3.98,6.31$ & $10,12.6,15.8,20,25.1,31.6$ \\
& 10 & $7.9,10,12.6,15.8,20,25.1$, \\
158 & $2.5,3.98$ & $5,6.3,7.9,10,12.6,15.8$ \\
& $6.31,10$ & $4,5,6.3,7.9,10,12.6$ \\
251 & $2.5,3.98,6.31,10$ & $2.5,3.2,4,5,6.3,7.9$ \\
398 & $2.5,3.98$ & $2.5,3.2,4,5,6.3,7.9$ \\
& $6.31,10$ & $2,2.5,3.2,4,5,6.3$ \\
\hline
\end{tabular}


Participants took a one-minute break every 5 min. Additionally, each participant took a rest of at least $30 \mathrm{~min}$ after 30 min of experiments.

\section{Results and discussion}

Detection of tactile beats (Experiment 1)

In this experiment, we examined the tactile beats phenomenon with a discrimination test to compare the standard vibration and comparison vibration that stimulated two tactile pins on the skin at each spatial interval. In condition 3, all of the trials were rated "same." In condition 1, 99.8\% of the trials were rated "same." This means that participants sensed the same stimulation between the standard vibration ( $250 \mathrm{~Hz}$ sinusoidal external stimulation) and the comparison vibration ( $254 \mathrm{~Hz}$ sinusoidal external stimulation). The results of condition 1 were expected because Pongrac shows that the resulting JND was $20 \%$ for vibrations of $250 \mathrm{~Hz}$, and much other research has shown through experimental results of vibrotactile discriminative increment or just noticeable difference (JND) that $4 \mathrm{~Hz}$ of difference around $250 \mathrm{~Hz}$ is not a noticeable difference in human tactile sensation (Goff 1967; Mahns et al. 2006; Pongrac 2008). From the result of condition 1 , we proved that it is perceptually difficult to detect the stimulus difference between 250 and $254 \mathrm{~Hz}$. However, subjects rated "different" for all the trials in condition 2 . This means that all participants correctly perceived and discriminated the $4 \mathrm{~Hz}$ change of a pin when another pin vibrates without a frequency change, although the amount of total intensity change in condition 2 was less than the change in condition 1 in the aspect of the physical parameter in each pin. Stimulating two vibrations with slight frequency differences simultaneously induced interference, which caused a new tactile perception like pitch variation based on the beats phenomenon. When the two tactile pins stimulated the skin, the vibrations were summed in the skin. The results of Experiment 1 show that slight frequency changes of one pin caused a large sensation variation although the same amount of frequency change to both two pins did not cause any perceptual variation.

From these results of Experiment 1, we concluded that it is easier to recognize a stimulus change between standard and comparison vibration when only one tactile pin changes frequency more slightly (condition 2) than when both pins changes frequency (condition 1). Additionally, the mean response is 3.72 to feel impacts from four envelope peaks of stimuli. These results mean that all subjects sensed the low frequency stimulus when two tactile pins, which have the distance of $1 \mathrm{~mm}$ from each other, are stimulated with slightly different sinusoidal frequencies. The stimulus of each pin was transferred to the mechanoreceptors according to the mechanical properties of the skin (Azañón and Soto-Faraco 2008). The results show that two vibrations with slight frequency differences induce low frequency sensation, which results from the interference between different harmonic motions with slightly different frequencies based on the beats phenomenon. Tactile beats cause the intensity change of stimulus temporally at the location of mechanoreceptors (Bensmaia et al. 2005). Participants could not detect all four peaks because of the limitations in tactile temporal numerosity judgment. Philippi tested temporal numerosity judgment for ranges from 2 to 10 pulses per second (Philippi et al. 2008). When we compare these data, it appears to be consistent that the four events in a second are underestimated at the tactile sense. Lechelt (1975) and Philippi et al. (2008) confirmed the general tendency toward underestimation with the amount of underestimation increasing with the increase in the number of pulse and the decrease in the interstimulus interval. Gallace et al. (2008) showed that tactile stimuli cannot be subitized when multiple tactile stimuli were simultaneously presented across the body. From their results, four events in a second are likely to fall into the "counting" region along the subitizing-counting-estimating continuum in tactile numerosity judgment in the temporal domain since it is not easy to discriminate four events in a second in all trials.

Our observation is related to the research by von Bekesy $(1957,1959)$ who has shown that the presence of beats phenomenon on the forearm can be perceived with two slightly different vibrations by using a vibrating coil of a loudspeaker unit. In this experiment, we try to observe the beats phenomenon and its characteristics at the finger pad under precisely controlled conditions. We measure whether the beats phenomenon can be perceived with small contactors (1 $\mathrm{mm}$ diameter) and $1 \mathrm{~mm}$ distance. We tested our idea with a piezoelectric bimorph to generate exact and accurate waveforms and measured the amplitude with a laser doppler vibrometer.

\section{Threshold of tactile beats (Experiment 2)}

In this experiment, we measured the amplitude threshold to perceive the beats sensation when two indentation pins that have small contactors (diameter $1 \mathrm{~mm}$ ) stimulate within a distance of $1 \mathrm{~mm}$ from each other. From the psychometric function curve, the beats detection threshold was defined as the vibration amplitude at which the subject gave "different" responses to $75 \%$ of stimulus deliveries. Figure $3 \mathrm{a}$ shows the psychometric functions for these five frequencies $(63.1,100,158,251$, and $398 \mathrm{~Hz})$ at each beats frequency $(2.5,3.98,6.31$, and $10 \mathrm{~Hz})$.

The mean change of the beats amplitude threshold according to standard frequency was obtained for 12 
Fig. 3 a The psychometric function curves relating to the discrimination rate and the amplitude between the standard vibration and comparison vibration for a series of five different frequencies. The psychometric curves are plotted with beats frequencies at 2.5, 3.98, 6.31, and $10 \mathrm{~Hz}$. The horizontal dotted lines represent $75 \%$ correct performance at which thresholds were estimated. b Beats detection threshold for two pin stimuli on the finger pad with the same amplitude
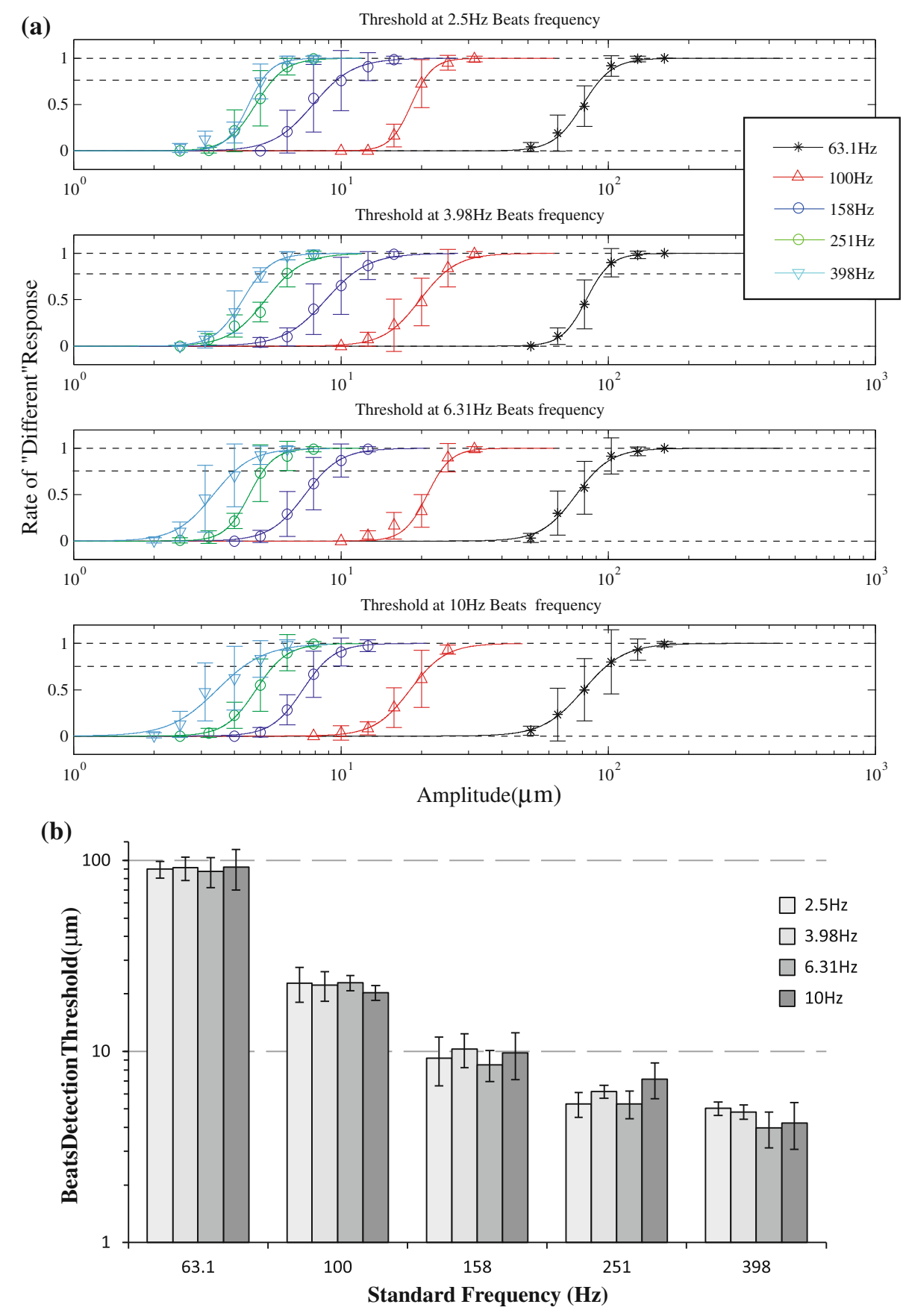

subjects from each psychometric curve. The beats detection threshold at each standard and beats frequency is shown in Table 2. For each beats stimulus, we averaged the graphs of beats detection thresholds across subjects at each standard and beats frequencies. The results are shown in Fig. 3b, with the error bars representing standard errors.

A repeated-measure ANOVA on the data from the full set of psychometric functions confirmed the significant effects of standard frequency for each beats frequency. We tested the effects of the two independent variables (standard frequency and beats frequency) to the threshold, which has proved that the standard frequency was statistically significant $[\mathrm{F}(4,44)=69.020, P<0.005]$, but the beats frequency did not $[\mathrm{F}(3,33)=0.825, P=0.490]$. The results show that the beats detection threshold decreases as the standard frequency increases.

Many research results show that the absolute vibrotactile detection threshold is a function of frequency (Bolanowski et al. 1994, 1988; Franzen 1969; Gescheider et al. 2002; Goble et al. 1996; Sherrick 1953; Verrillo 1963). From the previous work (Kyung et al. 2005), we investigated the absolute detection threshold with tactile pins of the small-area 
Table 2 Mean and variance of beats detection amplitude threshold $(\mu \mathrm{m})$ and steepness

\begin{tabular}{|c|c|c|c|c|c|c|}
\hline \multicolumn{2}{|c|}{ Envelop frequency $(\mathrm{Hz})$} & \multicolumn{5}{|c|}{ Standard frequency (Carrier frequency) $(\mathrm{Hz})$} \\
\hline & & 63.1 & 100 & 158 & 251 & 398 \\
\hline \multirow[t]{2}{*}{2.5} & Mean & $89.83 \pm 8.87$ & $22.82 \pm 4.77$ & $9.24 \pm 2.62$ & $5.32 \pm 0.79$ & $5.03 \pm 0.40$ \\
\hline & Steepness & 1.116 & 1.075 & 10,103 & 1.094 & 1.100 \\
\hline \multirow[t]{2}{*}{3.98} & Mean & $91.36 \pm 12.79$ & $22.23 \pm 3.90$ & $10.28 \pm 2.05$ & $6.17 \pm 0.47$ & $4.83 \pm 0.43$ \\
\hline & Steepness & 1.080 & 1.108 & 1.132 & 1.163 & 1.116 \\
\hline \multirow[t]{2}{*}{6.31} & Mean & $87.65 \pm 15.52$ & $22.89 \pm 2.14$ & $8.53 \pm 1.56$ & $5.32 \pm 0.88$ & $3.98 \pm 0.85$ \\
\hline & Steepness & 1.114 & 1.101 & 1.127 & 1.087 & 1.098 \\
\hline \multirow[t]{2}{*}{10} & Mean & $91.97 \pm 22.01$ & $20.36 \pm 1.83$ & $9.83 \pm 2.73$ & $7.17 \pm 1.51$ & $4.23 \pm 1.16$ \\
\hline & Steepness & 1.096 & 1.121 & 1.126 & 1.099 & 1.147 \\
\hline
\end{tabular}

tactor at several frequencies of sinusoidal input. The studies show that vibrotactile perception of a human finger becomes more sensitive as the vibration frequency of stimulation increases except at very high frequency ranges. And in the aspect of the beats detection threshold, we found that a human finger is more sensitive with increasing frequency. Furthermore, participants feel the similar thresholds at the same carrier frequency even though the beats frequency is different. From the relation, lower frequency feeling by tactile beats is mediated by the mechanoreceptor engaged with carrier frequency from the results.

In addition, our other concern is the relation between the "ratio of the absolute tactile stimulus detection threshold to beats stimulus detection threshold" and "standard frequency." To compare the absolute detection threshold (Kyung et al. 2006) and the beats detection threshold, we present the relation between human vibrotactile beats perception and frequency. Figure 4 shows the means of the ratio of the absolute vibration detection threshold to the beats detection threshold according to standard frequency, which proves that the ratio increases as the standard frequency increases. For the data between the ratio of the absolute vibration detection threshold to the beats detection threshold and standard frequency, there is a significant linear trend across the standard frequency $(\mathrm{F}(1,11)=104.738$, $P<0.005)$. The results show in order to recognize beats with two pins in the case of low standard frequency, a percipient needs high amplitude stimulus of two pins compared to the absolute vibration detection threshold. As the standard frequency increases, the required amplitude level at two pins of the beats signal compared to absolute vibration decreases for the detection of beats. It means that higher carrier frequency is more sensitive and easier to detect beats because of the lower absolute vibratory detection threshold and beats detection threshold. Beats detection is caused in the mechanoreceptors. Beats feeling is mediated by the mechanoreceptor engaged with carrier frequency. From the previous skin and mechanoreceptor

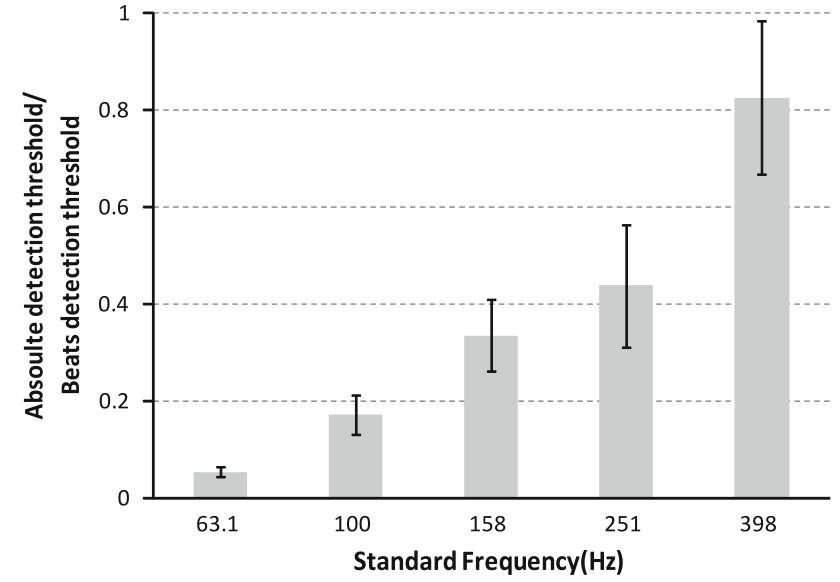

Fig. 4 Amplitude ratio of absolute tactile stimulus detection threshold to beats detection thresholds

model, action potential by the displacement of skin surface is generated through the skin and nerve (Pawluk and Howe 1997). Stimuli on the skin for beats generation are merged at the mechanoreceptor and generate action potentials. The stimuli are similar to amplitude modulation of vibration with carrier frequency at the mechanoreceptors. As the standard frequency increases, the beats detection threshold decreases because the absolute detection threshold is lower at the higher frequency.

\section{Conclusion}

In perception, two vibrations with slightly different frequencies induce the beats phenomenon. The present study finds that subjects can perceive beats with two tactile stimuli in different locations at the same time with different frequencies using small contactors. The alternating constructive and destructive interference between the two pins' tactile stimulation of the skin causes a lower frequency tactile sensation based on the beats phenomenon on the skin. 
The present study examined the tactile beats detection threshold on the index finger when two stimuli $1 \mathrm{~mm}$ distance apart from each other vibrated on the finger at the same time at slightly different sinusoidal frequencies (Fig. 2). Pawluk investigates the holistic model of human touch (Pawluk and Howe 1997). From the model, they show the mechanics of the skin, the mechanics of the end organ, the creation of a generator potential, the initiation of an action potential, and the branching structure of the afferent fibers. Simplified estimated lumped finger model is made to show how to change temporal and spatial displacement at the located pacinian in skin. From the model, the displacement of the skin surface causes the modulation of pacinian in the skin. It causes conduction of the nerve fiber by the Hodgkin-Huxley model. From their research, they conclude that two and more stimuli could sum at the mechanoreceptors because skin and end organ have mechanical properties. We present two stimuli on the skin surface. The stimuli are merged at the pacinian in the skin and slightly different frequency generated beats stimuli. We present the relation between standard frequencies and amplitudes of two vibrations, which stimulate with slightly different frequencies in different locations at the same time by generating beats sensation. The elevation of the beats detection threshold with two tactile pins vibrating at slightly different frequencies on the skin may be associated with the decreasing standard frequency of the tactile pins (Fig. 3a). In addition, the ratio of the absolute threshold to the beats detection threshold shows that beats are easier to perceive at a higher frequency when the stimulus is detected (Fig. 4). These results conclude that human tactile beats perception becomes more sensitive as the vibration frequency of stimulation increases.

Open Access This article is distributed under the terms of the Creative Commons Attribution Noncommercial License which permits any noncommercial use, distribution, and reproduction in any medium, provided the original author(s) and source are credited.

\section{References}

Azañón E, Soto-Faraco S (2008) Changing reference frames during the encoding of tactile events. Curr Biol 18(14):1044-1049

Bensmaia S, Hollins M, Yau J (2005) Vibrotactile intensity and frequency information in the pacinian system: a psychophysical model. Percept Psychophys 67(5):828-841

Bolanowski SJ Jr, Gescheider GA, Verrillo RT, Checkosky CM (1988) Four channels mediate the mechanical aspects of touch. J Acoust Soc Am 84(5):1680-1694

Bolanowski SJ, Gescheider GA, Verrillo RT (1994) Hairy skin: psychophysical channels and their physiological substrates. Somatosens Mot Res 11(3):279-290

Dinse HR, Kleibel N, Kalisch T, Ragert P, Wilimzig C, Tegenthoff M (2006) Tactile coactivation resets age-related decline of human tactile discrimination. Ann Neurol 60(1):88-94
Franzen O (1969) The dependence of vibrotactile threshold and magnitude functions on stimulation frequency and signal level. A perceptual and neural comparison. Scand J Psychol 10(4):289-298

Frisken-Gibson SF, Bach-y-Rita P, Tompkins WJ, Webster JG (1987) A 64-solenoid, four-level fingertip search display for the blind. IEEE Trans Biomed Eng 34(12):963-965

Gallace A, Tan HZ, Spence C (2008) Can tactile stimuli be subitised? An unresolved controversy within the literature on numerosity judgments. Perception 37(5):782-800

Gescheider GA, Bolanowski SJ, Pope JV, Verrillo RT (2002) A fourchannel analysis of the tactile sensitivity of the fingertip: frequency selectivity, spatial summation, and temporal summation. Somatosens Mot Res 19(2):114-124. doi:10.1080/0899022 0220131505

Goble AK, Collins AA, Cholewiak RW (1996) Vibrotactile threshold in young and old observers: the effects of spatial summation and the presence of a rigid surround. J Acoust Soc Am 99(4 Pt 1):2256-2269

Goff GD (1967) Differential discrimination of frequency of cutaneous mechanical vibration. J Exp Psychol 74(2):294-299

Hayward V, Cruz-Hernandez JM (2000) Tactile display device using distributed lateral skin stretch. Adv Robot Haptics 2000:13091314

Killebrew JH, Bensmaia SJ, Dammann JF, Denchev P, Hsiao SS, Craig JC, Johnson KO (2007) A dense array stimulator to generate arbitrary spatio-temporal tactile stimuli. J Neurosci Methods 161(1):62-74

Kyung K-U, Ahn M, Kwon D-S, Srinivasan MA (2005) A compact broadband tactile display and its effectiveness in the display of tactile form. Haptic interfaces for virtual environment and teleoperator systems, 2005 WHC 2005. First joint eurohaptics conference and symposium on 2005. pp 600-601

Kyung K-U, Ahn M, Kwon D-S, Srinivasan MA (2006) A compact planar distributed tactile display and effects of frequency on texture judgment. Adv Robotics 20:563-580

Kyung KU, Kim SC, Kwon DS (2007) Texture display mouse: vibrotactile pattern and roughness display. IEEE/ASME Trans Mechatron 12(3):356-360

Lechelt EC (1975) Temporal numerosity discrimination: intermodal comparisons revisited. Br J Psychol 66(1):101-108

Lim S-C, Kim S-C, Hwang J-H, Kwon D-S (2010) Physical contact of devices: utilization of beats for interpersonal communication. In: Kappers A, van Erp J, Bergmann Tiest W, van der Helm F (eds) Haptics: generating and perceiving tangible sensations, vol 6191. Lecture notes in computer science. Springer, Heidelberg, pp 348 353. doi:10.1007/978-3-642-14064-8_50

Lim S-C, Kyung K-U, Kwon D-S (2011) Presentation of surface height profiles based on frequency modulation at constant amplitude using vibrotactile elements. Adv Robot 25(16):2065-2081

Mahns DA, Perkins NM, Sahai V, Robinson L, Rowe MJ (2006) Vibrotactile frequency discrimination in human hairy skin. J Neurophys 95(3):1442-1450. doi:10.1152/jn.00483.2005

Makino Y, Maeno T, Shinoda H (2011) Perceptual characteristic of multi-spectral vibrations beyond the human perceivable frequency range. In: World haptics conference (WHC), 2011 IEEE, 21-24 June 2011, pp 439-443

Manning H, Tremblay F (2006) Age differences in tactile pattern recognition at the fingertip. Somatosens Mot Res 23(3): 147-155

Medina J, Rapp B (2008) Phantom tactile sensations modulated by body position. Curr Biol 18(24):1937-1942

Oyarzabal M, Nakatani M, Howe RD (2007) Vibration enhances geometry perception with tactile shape displays. In: Paper presented at the proceedings of the second joint EuroHaptics conference and symposium on haptic interfaces for virtual environment and teleoperator systems, Tsukuba, Japan 
Park G, Choi S (2011) Perceptual space of amplitude-modulated vibrotactile stimuli. In: World Haptics Conference (WHC), 2011 IEEE, 21-24 June 2011, pp 59-64

Pawluk DTV, Howe RD (1997) A holistic model of human touch. Annu Comput Neurosci Meet 1997:759-764

Philippi TG, van Erp JBF, Werkhoven PJ (2008) Multisensory temporal numerosity judgment. Brain Res 1242:116-125

Pongrac H (2008) Vibrotactile perception: examining the coding of vibrations and the just noticeable difference under various conditions. Multime'd Syst 13(4):297-307

Rao SS (1995) Mechanical vibrations, 3rd edn. Prentice Hall, pp 53-55
Sherrick CE (1953) Variables affecting sensitivity of the human skin to mechanical vibration. J Exp Psychol 45(5):273-282

Verrillo RT (1963) Effect of contactor area on the vibrotactile threshold. J Acoust Soc Am 35(12):1962-1966

von Bekesy G (1957) Sensations on the skin similar to directional hearing, beats, and harmonics of the ear. J Acoust Soc Am 29(4):489_ 501

von Bekesy G (1959) Synchronism of neural discharges and their demultiplication in pitch perception on the skin and in hearing. J Acoust Soc Am 31(3):338-349 